\title{
Optimal Polygonal Representation of Planar Graphs
}

\author{
C. A. Duncan · E. R. Gansner • Y. F. Hu • \\ M. Kaufmann • S. G. Kobourov
}

Received: April, 2011

\begin{abstract}
In this paper, we consider the problem of representing graphs by polygons whose sides touch. We show that at least six sides per polygon are necessary by constructing a class of planar graphs that cannot be represented by pentagons. We also show that the lower bound of six sides is matched by an upper bound of six sides with a linear-time algorithm for representing any planar graph by touching hexagons. Moreover, our algorithm produces convex polygons with edges having at most three slopes and with all vertices lying on an $O(n) \times O(n)$ grid.
\end{abstract}

Keywords Planar graphs · Contact graphs · Graph drawing · Polygonal drawings

\section{Introduction}

For both theoretical and practical reasons, there is a large body of work considering how to represent planar graphs as contact graphs, i.e., graphs whose vertices are represented by geometrical objects with edges corresponding to two objects touching

A preliminary version of this paper appeared in LATIN 2010, Oaxaca, Mexico

C. A. Duncan

Dept. of Computer Science, Louisiana Tech University

E-mail: duncan@latech.edu

E. R. Gansner · Y. F. Hu

AT\&T Research Labs, Florham Park, NJ

E-mail: $\{$ erg, yifanhu\}@ research.att.com

M. Kaufmann

Wilhelm-Schickhard-Institut for Computer Science, Tübingen University

E-mail: mk@informatik.uni-tuebingen.de

S. G. Kobourov

Dept. of Computer Science, University of Arizona

Tel.: + 123-45-678910

Fax: +123-45-678910

E-mail: kobourov@cs.arizona.edu 


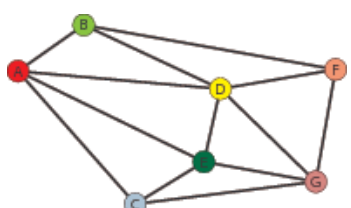

(a)

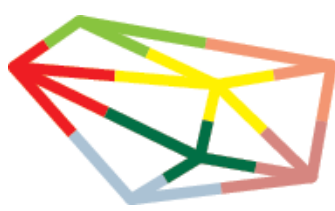

(b)

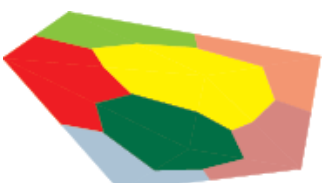

(c)

Fig. 1 (a) A drawing of a planar graph. (b) We apportion the edges to the endpoints by cutting each edge in half. (c) We then apportion the faces to form polygons.

in some specified fashion. Typical classes of objects might be curves, line segments or isothetic rectangles, and an early result is Koebe's theorem [23], which shows that all planar graphs can be represented by touching disks.

In this paper, we consider contact graphs whose objects are simple polygons, with an edge occurring whenever two polygons have non-trivially overlapping sides. As with treemaps [5], such representations are preferred in some contexts [6] over the standard node-link representations for displaying relational information. Using adjacency to represent a connection can be much more compelling, and cleaner, than drawing a line segment between two nodes. For ordinary users, this representation suggests the familiar metaphor of a geographical map.

It is clear that any graph represented this way must be planar. As noted by de Fraysseix et al. [8], it is also easy to see that all planar graphs have such representations for sufficiently general polygons. Starting with a straight-line planar drawing of a graph, we can create a polygon for each vertex by taking the midpoints of all adjacent edges and the centers of all neighboring faces. Note that the number of sides in each such polygon is proportional to the degree of its vertex. Moreover, these polygons are not necessarily convex; see Figure 1 .

It is desirable, for aesthetic, practical and cognitive reasons, to limit the complexity of the polygons involved, where "complexity" here means the number of sides in the polygon. Fewer sides, as well as wider angles in the polygons, make for simpler and cleaner drawings. In related applications such as floor-planning [27], physical constraints make polygons with very small angles or many sides undesirable. One is then led to consider how simple such representations can be. How many sides do we really need? Can we insist that the polygons be convex, perhaps with a lower bound on the size of the angles or the edges? If limiting some of these parameters prevents the drawing of all planar graphs, which ones can be drawn?

\subsection{Our Contribution}

This paper provides answers to some of these questions. Previously, it was known [15 27] that triangulated planar graphs can be represented using non-convex octagons. On the other hand, it is not hard to see that one cannot use triangles (e.g., $K_{5}$ minus one edge cannot be represented with triangles [12]).

Our main result is showing that hexagons are necessary and sufficient for representing all planar graphs. For necessity we construct a class of graphs that cannot be represented using five or fewer sides. For sufficiency, we prove the following: 
Theorem 1 For any planar graph $G$ on $n$ vertices, we can construct in linear time on an $O(n) \times O(n)$ grid a touching hexagons representation of $G$ with convex regions. Moreover, if the graph is a triangulation, the representation is also a tiling.

Note, if the input graph is not triangulated, there might be convex holes. We, in fact, prove this theorem using two different methods. First, in Sections 3 and 4 , we describe a linear-time algorithm that produces a representation using convex hexagons along with a linear-time compaction algorithm to reduce the initial exponential area to an $O(n) \times O(n)$ integer grid. Second, in Section 5 , we show how modifying Kant's algorithm for hexagonal grid drawings of 3-connected, 3-regular planar graphs [20] produces a similar result by different means. In both variations, the drawings use at most three slopes for the sides, for example, 1, 0 and -1 .

\subsection{Related Work}

As remarked above, there is a rich literature related to various types of contact graphs. There are many results considering curves and line segments as objects (cf. [16, 17]). For closed shapes such as polygons, results are rarer, except for axis-aligned (or isothetic) rectangles. In a sense, results on representing planar graphs as "contact systems" can be dated back to Koebe's 1936 theorem [23] which states that any planar graph can be represented as a contact graph of disks in the plane.

The focus of this paper is side-to-side contact of polygons. The algorithms of He [15] and Liao et al. [27] produce contact graphs of this type for triangulated graphs, with nodes represented by the union of at most two isothetic rectangles, thus giving a polygonal representation by non-convex octagons.

We now turn to contact graphs using isothetic rectangles, which are often referred to as rectangular layouts. This is the most extensively studied class of contact graphs, due in part to its relation to application areas such as VLSI floor-planning [25],34], architectural design [30] and geographic information systems [11], but also due to the mathematical ramifications and connections to other areas such as rectangle-ofinfluence drawings [28] and proximity drawings [2, 19].

Graphs allowing rectangular layouts have been fully characterized [29,32] with linear algorithms for deciding if a rectangular layout is possible and, if so, constructing one. The simplest formulation [6] notes that a graph has a rectangular layout if and only if it has a planar embedding with no filled triangles. Thus, $K_{4}$ has no rectangular layout. Buchsbaum et al. [6] also show, using results of Biedl et al. [4], that graphs that admit rectangular layouts are precisely those that admit a weaker variation of planar rectangle-of-influence drawings.

Rectangular layouts required to form a partition of a rectangle are known as rectangular duals. In a sense, these are "maximal" rectangular layouts; many of the results concerning rectangular layouts are built on results concerning rectangular duals. Graphs admitting rectangular duals have been characterized [14,24,26] and there are linear-time algorithms [14,22] for constructing them.

Another view of rectangular layouts arises in VLSI floor-planning, where a rectangle is partitioned into rectilinear regions so that region adjacencies correspond to a given planar graph. It is natural to try to minimize the complexities of the resulting regions. The best known results are due to He [15] and Liao et al. [27] who show that 
regions need not have more than 8 sides. Both of these algorithms run in $O(n)$ time and produce layouts on an $O(n) \times O(n)$ integer grid where $n$ is the number of vertices.

Rectilinear cartograms can be defined as rectilinear contact graphs for vertexweighted planar graphs, where the area of a rectilinear region must be proportional to the weight of its corresponding node. Even with this extra condition, de Berg et al. [3] show that rectilinear cartograms can always be constructed in $O(n \log n)$ time, using regions having at most 40 sides. The resulting regions, however, are highly non-convex and can have poor aspect ratio. Recently, Alam et al. [1] explore optimal bounds on the complexity of the polygons needed to produce point-contact and side-contact representations of subclasses of vertex-weighted planar graphs with additional restrictions such as convexity and hole-free regions.

Although not considered by the authors, an upper bound of six for the minimum number of sides in a touching polygon representation of planar graphs might be obtained from the vertex-to-side triangle contact graphs of de Fraysseix et al. [8]. The top edge of each triangle can be converted into a raised 3-segment polyline, clipping the tips of the triangles touching it from above, thereby turning the triangles into side-touching hexagons. This approach might prove difficult for generating hexagonal representations as it involves computing the amounts by which each triangle may be raised so as to become a hexagon without changing any of the adjacencies. Moreover, the nature of such an algorithm would produce many "holes," potentially making such drawings less appealing, or requiring further modifications. In [13], Gonçalves et al. describe a similar approach after presenting an algorithm to create primal-dual triangle contact representations, where each node and face are represented as triangles.

\subsection{Preliminaries}

Touching Hexagons Graph Representation: Throughout this paper, we assume we are dealing with a connected planar graph $G=(V, E)$. We would like to construct a set of closed simple polygons $R$ whose interiors are pairwise disjoint, along with an isomorphism $\mathcal{R}: V \rightarrow R$, such that for any two vertices $u, v \in V$, the boundaries of $\mathcal{R}(u)$ and $\mathcal{R}(v)$ overlap non-trivially if and only if $\{u, v\} \in E$. For simplicity, we adopt a convention of the cartogram community and define the complexity of a polygonal region as the number of sides it has. We call the set of all graphs having such a representation where each polygon in $R$ has complexity 6 touching hexagons graphs.

Canonical Labeling: Our algorithms begin by first computing a planar embedding of the input graph $G=(V, E)$ and using that to obtain a canonical labeling of the vertices. A planar embedding of a graph is simply a clockwise order of the neighbors of each vertex in the graph. Obtaining a planar embedding can be done in linear time using the algorithm by Hopcroft and Tarjan [18]. The canonical labeling or order of the vertices of a planar graph was defined by de Fraysseix et al. [10] in the context of straight-line drawings of planar graphs on an integer grid of size $O(n) \times O(n)$. While the first algorithm for computing canonical orders required $O(n \log n)$ time [9], Chrobak and Payne [7] have shown that this can be done in $O(n)$ time.

In this section we review the canonical labeling of a planar graph as defined by de Fraysseix et al. [9]. Let $G=(V, E)$ be a fully triangulated planar graph embedded in 
the plane with exterior face $u, v, w$. A canonical labeling of the vertices $v_{0}=u, v_{1}=$ $v, v_{2}, \ldots, v_{n-1}=w$ is one that meets the following criteria for every $2<i<n$ :

1. The subgraph $G_{i-1} \subseteq G$ induced by $v_{0}, v_{1}, \ldots, v_{i-1}$ is 2 -connected, and the boundary of its outer face is a cycle $C_{i-1}$ containing the edge $(u, v)$;

2. The vertex $v_{i}$ is in the exterior face of $G_{i-1}$, and its neighbors in $G_{i-1}$ form an (at least 2-element) subinterval of the path $C_{i-1}-(u, v)$.

The canonical labeling of a planar graph $G$ allows for the incremental placement of the vertices of $G$ on a grid of size $O(n) \times O(n)$ so that when the edges are drawn as straight-line segments there are no crossings in the drawing. The two criteria that define a canonical labeling are crucial for the region creation step of our algorithm.

Kant generalized the definition for triconnected graphs, partitioning the vertices into sets $V_{1}$ to $V_{K}$ that can be either singleton vertices or chains of vertices [21].

\section{Lower Bound of Six Sides}

In this section we show that at least six sides per polygon are sometimes needed in a touching polygons representation of a planar graph. We begin by constructing a class of planar graphs that cannot be represented by four-sided polygons and then extend the argument to show that the class also cannot be represented by five-sided polygons.

\subsection{Four Sides Are Not Enough}

Consider the fully triangulated graph $G^{k}$ in Figure 2 a). It has three nodes on the outer face $A, B$ and $C$, and contains a chain of nodes $1, \ldots, k$ which are all adjacent to $A$ and $B$. Consecutive nodes in the chain, $i$ and $i+1$, are also adjacent. The remaining nodes of $G^{k}$ are degree-3 nodes $l_{i}$ and $r_{i}$ inside the triangles $\Delta(A, i, i+1)$ and $\Delta(B, i, i+1)$.

Theorem 2 For $k \geq 33$, there does not exist a touching polygons representation for $G^{k}$ in which all regions have complexity four or less.

Proof Assume, for the sake of contradiction, that we are given a touching polygons drawing for $G^{k}$ in which all regions have complexity four or less. Without loss of generality, we assume that the drawing has an embedding that corresponds to the one shown in Figure 2a). Let $Q_{A}, Q_{B}$ and $Q_{C}$ denote the quadrilaterals representing nodes $A, B$ and $C$, and let $Q_{i}$ denote the quadrilateral representing node $i$. Once again, without loss of generality, let $Q_{A}$ lie in the left corner, $Q_{B}$ in the right corner and $Q_{C}$ at the top of the drawing.

We start with an observation.

Observation 1: Any corner of a quadrilateral can be adjacent to at most two disjoint quadrilaterals that (non-trivially) touch one of its sides. Since there are $c=8$ corners of $Q_{A}$ and $Q_{B}$, we have at most 16 quadrilaterals of the chain $Q_{1}, \ldots, Q_{k}$ that are adjacent to corners of $Q_{A}$ and/or $Q_{B}$.

We now consider the quadrilaterals that are not adjacent to any of these corners.

Let $Q_{i}$ be a quadrilateral that is not adjacent to any of the corners of the polygonal chains $A_{1}, A_{2}, A_{3}, A_{4}$ and $B_{1}, B_{2}, B_{3}, B_{4}$. Two of its corners are adjacent to the same 


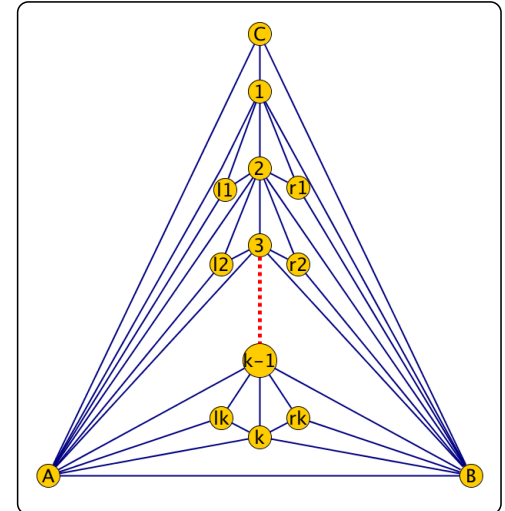

(a)

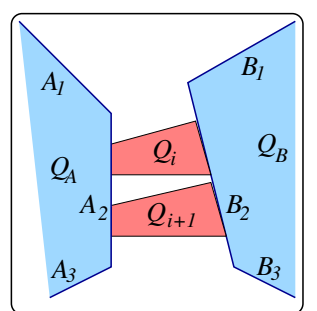

(b)

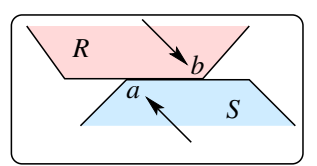

(c)

Fig. 2 (a) The graph $G^{k}$ that provides the counterexample. (b) A pair of subsequent fair quadrilaterals adjacent to the same sides of $Q_{A}$ and $Q_{B}$. (c) Illustration for Observation 2 shows one of three possible cases for two touching regions.

side $A_{p}$ of $Q_{A}$ and the other two are adjacent to the same side $B_{q}$ of $Q_{B}, 1 \leq p, q \leq 4$. We call such a quadrilateral a fair quadrilateral.

Lemma 1 For $k \geq 33$, in any touching quadrilaterals representation of $G^{k}$ there exists a pair of fair quadrilaterals $Q_{i}$ and $Q_{i+1}$ that are adjacent to the same sides of $Q_{A}$ and $Q_{B}$.

Proof We can partition the set of fair quadrilaterals into 16 equivalence classes $C_{p, q}$, $1 \leq p, q \leq 4$, that denote the sets of fair quadrilaterals that are adjacent to the same sides of $Q_{A}$ and $Q_{B}$. The equivalence class $C_{p, q}$ denotes that the pairs of sides $\left(A_{p}, B_{q}\right)$ are used.

Observe that if $Q_{i}$ is in an equivalence class $C$ and $Q_{i+1}$ is not fair, then since $Q_{i+1}$ must be adjacent to a corner, $Q_{i+2}$ cannot be in the equivalence class $C$. Thus, when we sweep through the chain of quadrilaterals $Q_{1}, \ldots, Q_{k}$, we simultaneously proceed through the equivalence classes. By the pigeonhole principle, if there are at least 17 fair quadrilaterals, then at least two of them must be in the same equivalence class. Combining that with the fact that there are at most 16 quadrilaterals that are not fair completes our proof.

Before continuing with the proof of Theorem 2, we include the following observation, partially illustrated in Figure 2 (c):

Observation 2: If there are two regions $R, S$ touching in some nontrivial interval $I=(a, b)$ then at $a$, there is a corner of $R$ or $S$. The same holds for corner $b$.

Using Observation 2, we see that each interval that is shared by two adjacent polygons ends at two of the corners of the two polygons. Now, let $\left(Q_{i}, Q_{i+1}\right)$ be a pair of fair same-sided quadrilaterals, touching sides $A_{p}$ and $B_{q}$. Since $Q_{i}$ is fair, the two corners associated with the adjacency of $Q_{A}$ must belong to $Q_{i}$ and the other two corners of $Q_{i}$ are associated with the adjacency with $Q_{B}$. The same applies for $Q_{i+1}$. 
Since $Q_{i}$ and $Q_{i+1}$ have to be adjacent, the two sides next to each other touch. From Observation 2, at least two corners of $Q_{i}$ or $Q_{i+1}$ are involved in the adjacency. For reference, label these two corners as $c_{1}$ and $c_{2}$. The quadrilateral $Q_{l_{i}}$, corresponding to node $l_{i}$, must touch quadrilaterals $Q_{A}, Q_{i}$ and $Q_{i+1}$. If $c_{1}$ (or $c_{2}$ ) were also associated with an adjacency to $Q_{A}$ then $Q_{l_{i}}$ could not be adjacent to all three quadrilaterals simultaneously. Therefore, $c_{1}$ and $c_{2}$ must correspond to adjacencies with $Q_{B}$. A similar argument for $Q_{r_{i}}$ shows that neither $c_{1}$ nor $c_{2}$ can correspond to adjacencies with $Q_{B}$ either. However, this is a contradiction as all four corners of both quadrilaterals are either associated with the adjacency with $Q_{A}$ or with $Q_{B}$.

\subsection{Five Sides Are Not Enough}

If we allow the regions to be pentagons, we must sharpen the argument a little more.

Lemma 2 For $k \geq 71$, in any touching pentagons representation for $G^{k}$, there exists a triple of fair pentagons $P_{i}, P_{i+1}, P_{i+2}$ adjacent to the same sides of $P_{A}$ and $P_{B}$.

Proof We prove this along the same lines as the proof for Lemma 1 As before, we can see that for a total of 10 corners, at most 20 pentagons of the inner chain are not fair. The number of equivalence classes of pentagons with sides solely on the same side of $P_{A}$ and $P_{B}$ is at most $5 \times 5=25$. Recall that pentagons belonging to the same equivalence class are sequential. Since we aim now for triples and not just for pairs, using the pigeonhole principle, if we have more than $2 \times 25=50$ fair pentagons at least three must belong to the same equivalence class. Therefore, as long as $k>20+50=70$, there exists a triple of fair same-sided pentagons.

Theorem 3 For $k \geq 71$, there does not exist a touching polygons representation for $G^{k}$ in which all regions have complexity five or less.

Proof From Lemma 2, let $\left(P_{i}, P_{i+1}, P_{i+2}\right)$ be a triple of fair same-sided pentagons, touching sides $A_{p}$ and $B_{q}$. From Observation 2, we know that each interval that is shared by two polygons ends at two of the corners of the two polygons. Consequently, four of the five corners for $P_{i}, P_{i+1}$ and $P_{i+2}$ are adjacent to $A_{p}$ or $B_{p}$. Since $P_{i}$ and $P_{i+1}$ have to be adjacent, the two sides next to each other touch. However, since there exist the polygonal regions representing $r_{i}$ and $l_{i}$, as before, the interval where $P_{i}$ and $P_{i+1}$ touch is disjoint from the regions $P_{A}$ and $P_{B}$. As each region can have at most five corners, four of which are adjacent to either $P_{A}$ or $P_{B}$, from Observation 2 we know that one corner from the adjacency with $P_{i}$ and $P_{i+1}$ belongs to $P_{i}$ and one belongs to $P_{i+1}$. Similarly, we know that the adjacencies of $r_{i+1}$ and $l_{i+1}$ imply that one corner of the adjacency of $P_{i+1}$ and $P_{i+2}$ belongs to $P_{i+1}$ and the other belongs to $P_{i+2}$. Due to planarity, we also know that $P_{i}$ and $P_{i+2}$ lie on opposite sides of $P_{i+1}$. As these corners cannot be adjacent to $P_{A}$ or $P_{B}$, we see that $P_{i+1}$ must have six distinct corners, two adjacent to $P_{A}$, two to $P_{B}$, one to $P_{i}$ and one to $P_{i+2}$, a contradiction.

Note that six-sided polygons are indeed sufficient to represent the graph in Figure 2(a). In particular, for fair polygons $P_{i}$ and $P_{i+1}$, we can use three segments on the lower side of $P_{i}$, while the upper side of $P_{i+1}$ consists of only one segment completely overlapping the middle of the three segments from the lower side of $P_{i}$. 


\section{Touching Hexagons Representation}

In this section, we present a linear-time algorithm that takes as input a planar graph $G=(V, E)$ and produces a representation of $G$ in which all regions are convex hexagons. This algorithm and the fact that every touching hexagons graph is necessarily planar proves that the class of planar graphs is equivalent to the class of touching hexagons graphs.

\subsection{Algorithm Overview}

We assume that the input graph $G=(V, E)$ is a fully triangulated planar graph with $|V|=n$ vertices. If the graph is planar but not fully triangulated, we can augment it to a fully triangulated graph with the help of dummy vertices and edges, run the algorithm below and remove the polygons that correspond to dummy vertices.

Traditionally, planar graphs are augmented to fully triangulated graphs by adding edges to each non-triangular face. Were we to take this approach, however, when we remove the dummy edges we would have to perturb the resulting space partition to remove polygonal adjacencies. As this is difficult to do, we convert our input graph to a fully triangulated one by adding one additional vertex to each face and connecting it to all vertices in that face. The above approach works if the input graph is biconnected. Singly-connected graphs must first be augmented to biconnected graphs as follows. Consider any articulation vertex $v$, and let $u$ and $w$ be consecutive neighbors of $v$ in separate biconnected components. Add a new vertex $z$ and the edges $(z, u)$ and $(z, w)$. Iterating for every articulation point biconnects $G$ and results in an embedding in which each face is bounded by a simple cycle. Since determining articulation points and adding vertices and edges to faces can be done in linear time, the augmentation step incurs only a linear amount of additional time to the main algorithm and adds at most a linear number of vertices and edges to the original graph.

The algorithm has two main phases. The first phase computes the canonical labeling. In the second phase we create regions with slopes $0,1,-1$ out of an initial isosceles right-angle triangle, by processing vertices in the canonical order. Each time a new vertex is processed, a new region is carved out of one or more already existing regions. At the end of the second phase of the algorithm we have a right-angle isosceles triangle that has been partitioned into exactly $n=|V|$ convex regions, each with at most 6 sides. We show that creating and maintaining the regions requires linear time in the size of the input graph. We illustrate the algorithm with an example; see Figure 3

\subsection{Region Creation}

In this section we describe the $n$-step incremental process of inserting new regions in the order given by the canonical labeling, where $n=|V|$. The regions will be carved out of an initial triangle with coordinates $(0,0),(-1,1),(1,1)$. The process begins by the creation of $R_{0}, R_{1}$, and $R_{2}$, which correspond to the first three vertices, $v_{0}, v_{1}, v_{2}$; see Figure 3 a). Note that the first three vertices in the canonical order form a triangular face in $G$ and hence must be represented as mutually touching regions. 


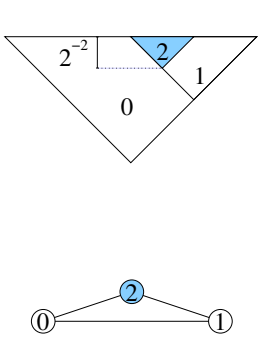

(a)
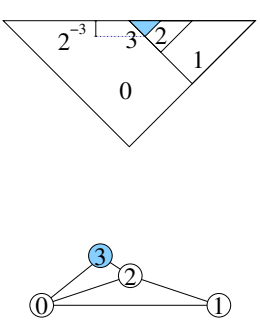

(b)
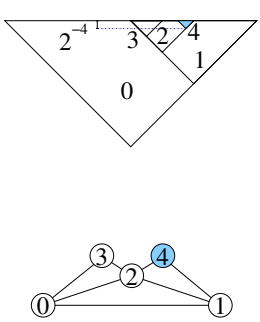

(c)
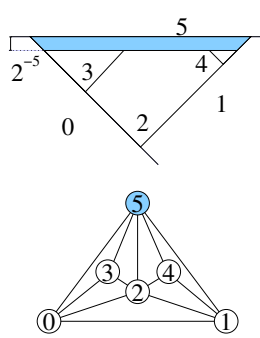

(d)

Fig. 3 Incremental construction of the touching hexagons representation of a graph. Shaded vertices on the bottom row and shaded regions on the top row are processed at each step. In general, the region defined at step $i$ is carved at distance $2^{-i}$ from the active front on the top. Note that the top row forms a horizontal line at all times.

At step $i$ of this process, where $2<i<n$, region $R_{i}$ will be carved out from the current set of regions. Define a region as "active" at step $i$ if it corresponds to a vertex that has not yet been connected to all its neighbors. An invariant of the algorithm is that all active regions are non-trivially tangent to the top side of the initial triangle, which we refer to as the "active front."

By criterion 2 of the canonical labeling and the active regions invariant, the current node $v_{i}$ is connected to two or more consecutive vertices on the outer face of $G_{i-1}$ and consecutive regions on the active front. Let $v_{a}$ and $v_{b}$ be the leftmost and rightmost neighbors of $v_{i}$ on the outer face with corresponding (active front) faces $R_{a}$ and $R_{b}$. The new region $R_{i}$ is defined to be an isosceles trapezoid formed by carving a horizontal line segment that is at distance $1 / 2^{i}$ from the active front and intersects the right side of $R_{a}$ and the left side of $R_{b}$. The left (respectively, right) side of the trapezoid has slope -1 (respectively, +1 ). If the right side of $R_{a}$ has slope +1 , a portion of its region is necessarily carved out by $R_{i}$. The same applies if the left side of $R_{b}$ has slope -1 . The regions between $R_{a}$ and $R_{b}$ have their upper segment carved and no longer being tangential to the active front are removed from the set of active regions. In addition, $R_{i}$ is added to the list of active regions. In Figure $3(\mathrm{~d})$, for example, both $R_{0}$ and $R_{1}$ have appropriate slopes and so are not carved and $R_{2}, R_{3}$, and $R_{4}$ are all removed from the active front.

Note, that if $d_{G_{i}}\left(v_{i}\right)=2$, then the length of the horizontal segment is 0 and the shape is an isosceles triangle. In this case, the geometry is such that exactly one of $R_{a}$ or $R_{b}$ must necessarily be carved. See Figures $3(\mathrm{a}-\mathrm{c})$.

Lemma 3 The above algorithm produces convex regions with at most 6 sides.

Proof The convexity of the regions is obvious from the fact that regions are created by a (linear) partitioning cut of a previous convex shape. Note that the above algorithm leads to the creation of at most ten different types of regions; see Figure 4 Each region has a horizontal top segment, a horizontal bottom segment (possibly of length 0 ), and sides with slopes -1 or 1 . Moreover, each region can be characterized as either opening (the first two in top row), static (the next four in the middle row), or closing (the last four in the bottom row), depending on the angles of the two sides 


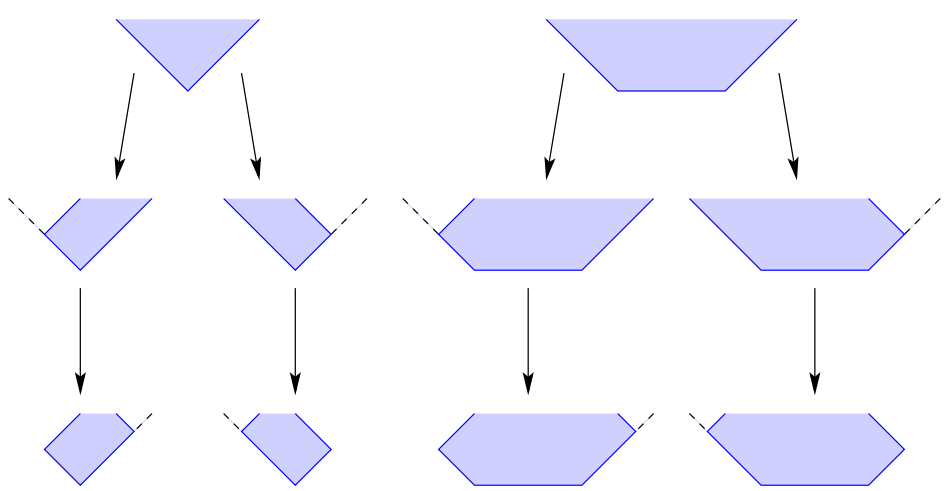

Fig. 4 There are a ten possible region shapes, falling into three categories: 2 opening, 4 static, and 4 closing. The arrows indicate carvings from one region to another.

connecting it to the top horizontal segment. At each iteration, each new region $R_{i}$ is an opening region. In the new region's creation, all affected regions except for $R_{a}$ and $R_{b}$ are carved with a horizontal line segment lying just below the top segment thus having no effect on the shapes of these regions and removing them from the active front. Consequently, the only new region shapes possible stem from cutting $R_{a}$ and $R_{b}$ when necessary with a slope -1 or +1 line respectively. As the lower vertex formed by each cut is at least half the distance to the active front from the previous vertices (those not on the active front), the only possible shapes are those shown in Figure 4 Observe that closing regions cannot be carved at all.

\subsection{Running Time}

The above algorithm can be implemented in linear time. The linear-time algorithm for computing a canonical labeling of a planar graph [7] requires a planar embedding as an input. Recall that a planar embedding of a graph is simply a clockwise order of the neighbors of each vertex in the graph. Obtaining a planar embedding can be done in linear time using the algorithm by Hopcroft and Tarjan [18].

Creating and maintaining the regions in the second phase of our algorithm can also be done in linear time. We next prove this by showing that each region requires $O(1)$ time to create and requires $O(1)$ number of modifications.

Consider the creation of new regions. From criterion 2 of the canonical labeling, when we process the current vertex $v_{i}$, it is adjacent to at least two consecutive vertices on the outer face of $G_{i-1}$. By construction of our algorithm the vertices in the outer face of $G_{i-1}$ correspond to active regions and so have a common horizontal tangent.

If $d_{G_{i}}\left(v_{i}\right)=2$, then a new region $R_{i}$ is carved out of one of the neighboring regions $R_{a}$ or $R_{b}$. Determining the coordinates of $R_{i}$ takes constant time, given the coordinates of $R_{a}$ and $R_{b}$ and the fact that $R_{i}$ will have height $1 / 2^{i}$ and will be tangent to the active frontier; see Figure 5 (a). When considering numerical precision, this exponential decrease in height could lead to $O(n)$ bits needed per vertex; however, as we show in Section 4 we only need the combinatorial structure and not the precise 


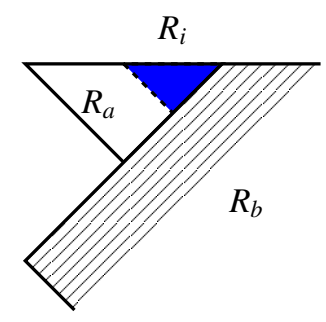

(a)

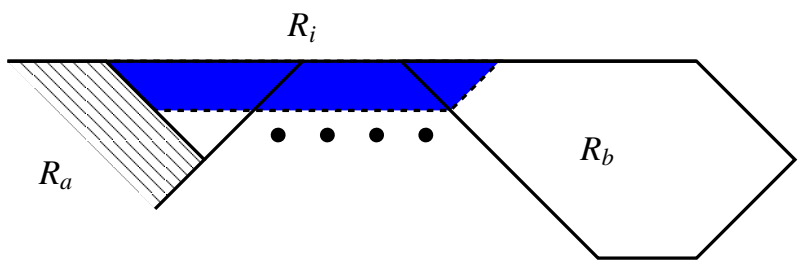

(b)

Fig. 5 Examples of introducing (the shaded) region $R_{i}$ between $R_{a}$ and $R_{b}$, (a) when $R_{i}$ is carved out of $R_{a}$ and $d_{G_{i}}\left(v_{i}\right)=2$, and (b) when $R_{i}$ is carved out of all neighbors of $v_{i}$ in $G_{i}$ except $R_{a}$.

geometric structure before proceeding to draw the regions in a more compact and efficient form.

If $d_{G_{i}}\left(v_{i}\right)>2$, then all regions between and possibly including $R_{a}$ and $R_{b}$ will have their corresponding regions carved, in order to create the new region $R_{i}$; see Figure 5 (b). In this case the coordinates of $R_{i}$ can also be determined in constant time given the coordinates of $R_{a}$ and $R_{b}$ and the fact that $R_{i}$ will have height $1 / 2^{i}$ and will be tangent to the active frontier.

Consider the modifications of existing regions. As can be seen from the hierarchy of regions on Figure 4, there are exactly 10 different kinds of regions and each region begins as either an isosceles trapezoid or triangle and undergoes at most three modifications including the final horizontal cut removing the region from the active front. Moreover, once a region goes from one type to the next, it can never change back to the same type. Finally note that the total number of region modifications is proportional to $|E|$ and since $G$ is planar, $|E|=O(|V|)$. Thus, each region needs at most a constant number of modifications from the time it is created to the end of the algorithm.

The algorithm described in this section, yields the following lemma:

Lemma 4 For any planar graph $G$ on $n$ vertices, we can construct in linear time a touching hexagons representation of $G$ with convex regions. Moreover, if the graph is a triangulation, the representation is also a tiling.

\section{Compaction Algorithm for Quadratic Area}

The algorithm given in Section 3.2 provides a touching hexagons representation of any planar graph. The incremental process carves out polygons within an ever smaller band of active front, therefore in practice the drawing is highly skewed, leading to exponential area. In this section we describe a compaction algorithm to get a drawing on an $O(n) \times O(n)$ grid.

When looking at the vertices and edges created in the algorithm for touching hexagons, if the horizontal edges are ignored, then the resulting graph is a "binary" tree, in the sense that each vertex has a degree of no more than 2. See Figure 6 


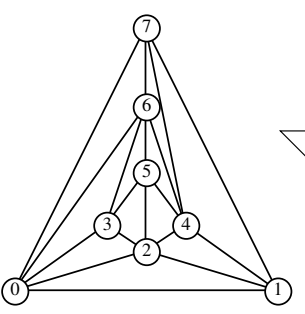

(a)

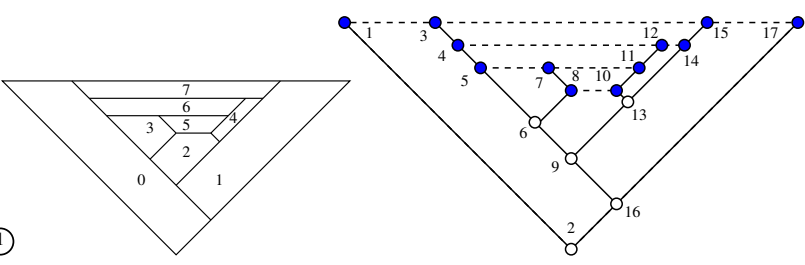

(b)

(c)

Fig. 6 An example of a capped binary tree. (a) The original graph. (b) The hexagonal representation (not drawn to scale to conserve space). (c) The corresponding capped binary tree with shaded nodes representing cap nodes and dashed lines representing capped sets. The nesting of all capped sets is $(1,3,(4,(5,7,(8,10), 11), 12,14), 15,17)$. We draw our binary tree upwards with the root at the bottom to correlate better with the hexagonal drawing algorithm.

From this observation, we can generalize the compaction problem to the tree drawing routine described below.

We start with some definitions. Order the nodes according to their inorder traversal. A cap set is an ordered subset of the nodes such that

1. The first (resp. last) node has exactly one child, the left (resp. right) child.

2. All other nodes are leaf nodes, with the addition that for the outermost cap the first and last nodes are also leaf nodes.

3. The ordering of nodes in the cap set follows the same inorder traversal ordering.

4. Any two cap sets are non-overlapping. However, one may be nested in another, in the sense that if one set $C$ goes from node $a$ to node $b$ and is contained in a second set $C^{\prime}$ then there exist two consecutive nodes $i, j$ in $C^{\prime}$ such that $i<a<b<j$.

Define a capped binary tree as a binary tree where every node either has two children (proper) or is assigned to a specific cap set. For convenience, we often refer to individual cap nodes in a cap set or to cap node pairs $(u, v)$ of neighboring (consecutive) cap nodes in a cap set.

Figure 6 illustrates the correlation to the hexagons created by the algorithm in Section 3.2 and provides an example of a capped binary tree, where the nodes in the tree represent the vertices formed in the drawing (not the hexagon faces), the edges are precisely the (non-horizontal) edges of the drawing, and each cap set is a maximal connected component of vertices and horizontal edges of the drawing.

The capped binary tree drawing problem is to take a capped binary tree and draw it on an integer grid such that 1) there are no edge crossings except at common endpoints; 2) each right (resp. left) edge is drawn with a slope of +1 (resp. -1); and 3) all nodes in a cap set are drawn with the same $y$-coordinate such that they can be connected by a horizontal line segment without crossing any other edges (except at the nodes in the set).

Before proving that we can draw capped binary trees on an $n / 2 \times n / 2$ grid, where $n$ is the number of nodes in the tree, we first present a divide-and-conquer compaction algorithm to accomplish this. The algorithm is inspired by the layered tree drawing algorithm [33] with the additional aforementioned constraints. 
Let $G_{T 6 G}$ be the graph derived from the touching hexagons algorithm, formed by taking the vertices as the intersections of the regions and the edges as (portions of) the sides of the regions connecting the vertices; see Figure 6 (c) where the edges are both the solid and dashed lines. Let $G_{T}$ be the corresponding capped binary tree, formed by removing the horizontal edges. Our compaction algorithm, described in detail below, proceeds by incrementally removing and placing a subset of the leaf nodes from a subtree $G_{c}$ initially set to $G_{T}$. For each of the leaf nodes of $G_{c}$ removed, the resulting placement requires the node to be connected to all of its child nodes (if any) in the original tree $G_{T}$ and also might require adjusting the position of one of its subtrees. Our process works by only adjusting the horizontal positions of any node, thus preserving a subtree's vertical position. For performance reasons, we actually delay the horizontal shifting by merely recording the shift needed for a subtree in its root. The shifts are then propagated through the tree in a final post-processing stage. Initially, the $x$ and $y$ positions as well as the horizontal shift of every node is set to 0 .

Before proceeding with the details of the algorithm, we clarify precisely those leaf nodes that are removed and placed at each iteration. Define the active front node set $F$ of $G_{c}$ as the maximum subset of leaf nodes of $G_{c}$, such that a cap node is in $F$ if and only if all the nodes in its cap set are also in $F$. The initial active front is precisely those vertices at the upper edge of the outer triangular region; see Figure 10

1. For each node $v$ in the active front node set $F$ of $G_{c}$,

a) if $v$ is a leaf in $G_{T}$, we do nothing ( $v$ remains at $(0,0)$ ).

b) if $v$ has one subtree in $G_{T}$ and if it is to the right, extend a slope +1 line from the root of this right subtree by 1 unit down and left to get the position of $v$. If it instead has a left child, extend a slope -1 line, down and right.

c) if $v$ has two subtrees in $G_{T}$, shift the right subtree horizontally so that the two subtrees have a "separation' 1 of either distance 1 or 2 , and the slope -1 (resp. +1 ) line from the root of the left (resp. right) subtree meet at a grid point, the assigned point for $v$. Record the shift used at the root of the right tree.

2. For each cap set $C$ in the front, set $h$ to be the maximum of the absolute values of $y$ coordinates of the cap nodes in $C$. For every cap node $v \in C$,

a) if $v$ is a leaf node in $G_{T}$, set $y(v)=-h$;

b) otherwise, by construction, node $v$ must have only one subtree. If it is to the right, extend the slope +1 line from the root of the subtree till it intersects with the line $y=-h$, and record the coordinates of the intersection point as the coordinates for $v$. If it is to the left, extend the slope -1 line instead.

3. Delete $F$ and its connecting edges from $G_{C}$, renaming the resulting tree $G_{C}$. If $G_{c}$ is not empty, go to Step 1.

4. Propagate the horizontal shifts from each node to its subtree via a pre-order traversal starting at the root of $G_{T}$ to obtain a final integer grid position for each node.

This algorithm yields a drawing of the $G_{T 6 G}$ on a grid. Figures $10-13$ illustrate execution of this algorithm on the graph from Figure 6 . Figure 14 shows some additional graphs, and their corresponding touching hexagons representations on a grid. Because the algorithm processes entire cap sets at a time, because Step 2 places all

1 We elaborate on what separation entails shortly. 


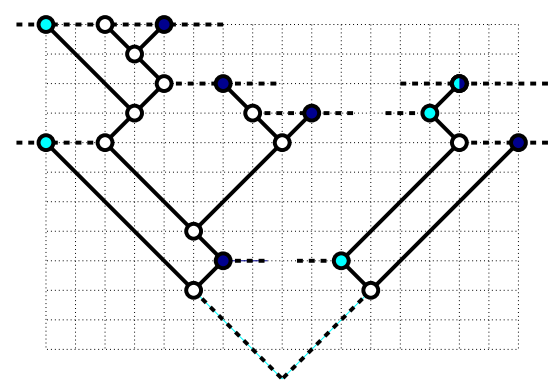

(a)

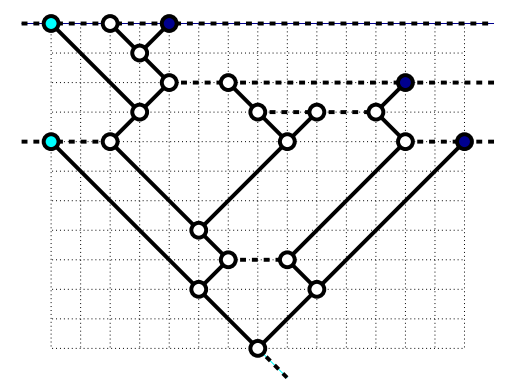

(b)

Fig. 7 An example of compacting two subtrees together during Step 1c (a) The two subtrees with left and right cap nodes shaded light and dark respectively. Observe that the top node in the right subtree is two-tone to reflect that it is both a left and right cap node. (b) The resulting tree after merging with the updated left and right cap nodes highlighted.

nodes in the same cap set at the same (lowest) height, and because it only shifts nodes horizontally, all cap nodes are drawn at the same vertical position. Further, because the algorithm also only connects the tree edges using line segments with slope \pm 1 and applies any horizontal shifts to the entire subtree via the final propagation step, all tree edges are drawn with slopes \pm 1 . Consequently, the drawing produced by this algorithm is a valid capped binary tree drawing.

We also need to show that the grid size used is reasonable. To bound the area, we must first elaborate on the compaction step (Step 1c) that combines two trees such that their separation is either distance 1 or 2 . This separation is not between the two roots of the subtrees but between the closest two nodes. In essence, we wish to compact the two subtrees as close as possible. Although there are several possible approaches including some that are more straightforward but slower than ours, we describe below a simple method to ensure that the algorithm's time remains linear.

For any subtree $T$, if we examine the cap nodes we see that some cap nodes are complete in the sense that all of the nodes in their cap set belong to $T$. We are interested in the cap sets that are not complete. A cap node is a left cap node of $T$ if it is the smallest (numbered) node in its cap set that is also in $T$ but not the smallest node in its entire cap set; essentially, the left side is not complete. We define a right cap node similarly. See Figure 7(a). Note that it is possible that a node is both a left and right cap node. Because of the fact that all non-cap nodes have two children and all left (respectively, right) cap nodes have only right (respectively, left) children if any, when joining two subtrees the two closest nodes will necessarily be between a left and right cap node (of the same cap set). We therefore maintain the list of left and right cap nodes and their respective offsets from the root.

Let $T\left(C_{L}\right)$ represent the set of left cap nodes and $T\left(C_{R}\right)$ represent the right cap nodes of subtree $T$. We maintain both sets as a doubly-connected circularly linked list ordered by their $y$-values. Each cap node maintains its relative $x$-distance from the node just before and after it in this list. In addition, we know the offset of the first cap node from the root. When merging two subtrees $T_{1}$ and $T_{2}$ where $T_{1}$ lies to the left of $T_{2}$, we can find the closest distance between them by starting with 
the first (right) cap node $r$ in the list $T_{1}\left(C_{R}\right)$, the one closest to the root and the first (left) cap node $l$ in the list $T_{2}\left(C_{L}\right)$. Observe that $r$ and $l$ must be in the same cap set. We then proceed to zip up the lists until the first one finishes, without loss of generality assume it is $T_{2}$. After each step, we can compute the offset of each cap node relative to its corresponding root and thus determine the closest we can bring the two subtrees. We can also maintain the list for the merged tree $T$ by setting $T\left(C_{L}\right)=T_{1}\left(C_{L}\right)$ and $T\left(C_{R}\right)=T_{2}\left(C_{R}\right)$ and then merging the remaining elements from $T_{1}\left(C_{R}\right)$ into $T\left(C_{R}\right)$. The same applies if $T_{1}$ finishes first (has the shorter list). Figure 7 (b) shows the resulting merge of two subtrees.

Lemma 5 Given any capped binary tree $T$, we can compute in linear time a capped binary tree drawing of $T$ on an $(n-1) \times(n-1) / 2$ grid.

Proof We have already described the linear-time algorithm that produces a valid compact drawing on an integer grid. However, it still remains to prove that the resulting drawing is sufficiently compact. We do this by inductively analyzing the separation between neighboring cap nodes, which are "joined" during the process described above. This proof is reminiscent of the one given by Kant [20].

For every cap node pair $(u, v)$, consecutive nodes in a cap set, let its interior cap set $C_{i}$ be the cap set (if one exists) whose first node is the next cap node in the inorder traversal of $T$ from $u$. Note that from the definition of the nesting of cap sets, the last node in $C_{i}$ would be the last cap node before $v$. If no such set exists, then let $C_{i}$ be $\{\operatorname{lca}(u, v)\}$, where lca $(\mathrm{u}, \mathrm{v})$ represents the least common ancestor of $u$ and $v$. If $u$ has a child node, it must be a left child and if $v$ has a child node, it must be a right child. Since any subtree necessarily has at least one cap node (a leaf of that subtree), this lca is also the only node in the inorder traversal between $u$ and $v$. We refer to $u$ and $v$ as the exterior cap nodes of $C_{i}$. Observe that the interior cap set will have its $y$-coordinate value closer to the root in the final drawing. For example, in Figure 6(c), the cap node pair $(4,12)$ has the interior cap set $\{5,7,11\}$, whereas the cap node pair $(12,14)$ has as the interior cap set the lca $\{13\}$.

Our proof uses the following inductive claim. After every iteration of our algorithm, for any subtree $T^{\prime}$ and any cap node pair $(u, v)$ in $T^{\prime}$ that is part of a cap set whose exterior cap nodes are not also in $T^{\prime}$ or is the outermost cap set, the (horizontal) distance between $(u, v)$ is no more than twice the number of cap node pairs in the inorder traversal of $T^{\prime}$, inclusive of the pair $(u, v)$.

If this claim holds, then the drawing is on an $(n-1) \times(n-1) / 2$ grid because the final drawing is a single tree with one row of cap nodes at the top whose width cannot exceed twice the number of cap node pairs in the tree. To bound the number of cap node pairs, notice that the graph formed by the binary tree edges combined with the horizontal (capping) edges forms a 3-regular graph, excluding the root and leftmost and rightmost vertices which have degree two. Since this graph has $(3 n-3) / 2$ edges and $n-1$ of the edges are tree edges, that leaves exactly $(n-1) / 2$ horizontal edges. Each horizontal edge corresponds to a unique cap node pair. The height follows from the \pm 1 slope of the non-horizontal edges.

Initially, every node is in its own subtree so the claim holds. Inspecting the algorithm reveals that the only place where the claim could change is in Step $1 \mathrm{c}$ where two subtrees $T_{1}$ and $T_{2}$ are merged. In addition, since the trees are simply shifted to 


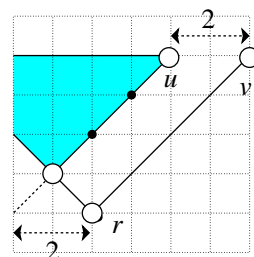

(a)

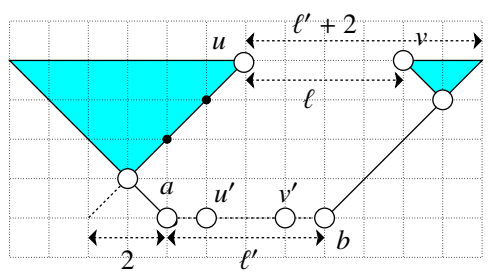

(b)

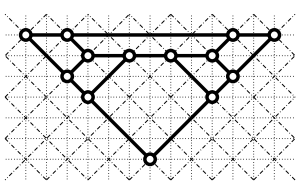

(c)

Fig. 8 Example of the merging of two subtrees during successive cap node pairs for (a) a single Ica node and $(u, v)$ and $(\mathrm{b})\left(u^{\prime}, v^{\prime}\right)$ and $(u, v)$. (c) A simple drawing of a capped binary tree highlighting both the normal grid (horizontal/vertical lines) and the rotated, space-efficient, grid (diagonal lines).

merge, the only possible change is due to the introduction of new cap node pairs, a cap node from each subtree is aligned with its neighbor in the other tree. In fact, since the merging process zips nested cap sets in succession, we are only concerned with the final width of the last cap pair merged. We again prove this by induction on the zipping process.

We claim that the width of the cap node pair $(u, v)$ merged is no more than twice the number of cap node pairs in its inorder traversal from $u$ to $v$. Let $(u, v)$ be the first cap node pair merged. Since the interior cap set of $(u, v)$ is simply $r$, which is the only node in the inorder traversal between $u$ and $v$, the resulting width at this stage is at most 2; see Figure 8 a). Thus, our claim holds after the first merged cap node pair.

We now progress inductively. Let $(u, v)$ be the next cap node pair merged with width $\ell,\left(u^{\prime}, v^{\prime}\right)$ be the previous pair, and $C$ be the interior cap set of $(u, v)$. Notice that $\left(u^{\prime}, v^{\prime}\right) \in C$. By induction, we know that the entire width $\ell^{\prime}$ of $C$ is no more than twice the number of cap node pairs in the inorder traversal from the first to last cap nodes in $C$. In addition, since $u$ has no right subtree and $v$ has no left subtree, the next cap node in the inorder traversal is the first cap node in $C$ and the last in the traversal is the last cap node in $C$. Therefore, we know that the number of cap node pairs in the inorder traversal from $u$ to $v$ is the same as the number for $C$ plus one, the one for $(u, v)$. Therefore, we need only to prove that $\ell \leq \ell^{\prime}+2$.

Let $a$ and $b$ be the first and last cap node in $C$; see Figure 8 (b). By the definition of a capped binary tree, we know that $a$ and $b$ each have only one child, a left and a right respectively. In addition, by Step 2 we know that one of the two child nodes is only one unit above its parent. Without loss of generality assume it is the left child of $a$. This means that the node is also one unit to the left of $a$. Node $u$ is a descendant of this left child but from the definition of the capped binary tree, $u$ can be found by traversing successive right children only. Therefore, the path from this left child to $u$ follows a straight line of slope +1 . This follows parallel with the line from $b$ through its right child. The (horizontal) distance from $u$ to this line is exactly $\ell^{\prime}+2$. Since the path from this right child to $v$ follows left children only (if any), the distance from $u$ to $v$ is $\ell \leq \ell^{\prime}+2$. This completes our proof.

For a clearer understanding and better symmetry with the construction technique used in Section 3, we used edges with slopes \pm 1 and 0 . We can improve the area bound slightly using a rotated drawing yielding the following corollary: 
Corollary 1 Given any capped binary tree $T$, we can compute in linear time a (rotated) capped binary tree drawing of $T$ on an $(n-1) / 2 \times(n-1) / 2$ grid.

Proof The trick is to rotate the grid $45^{\circ}$. The result is that the tree edges are drawn as horizontal and vertical lines while the "previously horizontal" capped edges are drawn with slope -1 . As Figure 8 (c) illustrates the only places where the vertices can lie from the initial drawing are the same as the overlaid rotated grid of dimension $(n-1) / 2 \times(n-1) / 2$. This can be further shown by observing that every vertex is connected to the root through a sequence of binary tree edges (of slope \pm 1 in the original grid). Thus, the original grid points lying in between the rotated grid squares cannot contain any vertices from the drawing. Thus, we have the same drawing on a slightly more compact grid.

Since the initial construction step does not need to create the hexagons explicitly, that step can be used simply to determine the combinatorial representation of the capped binary tree. This prevents any issues with numerical precision and representation. Combining Lemmas 4 and 5 yields our first proof for Theorem 1 .

\section{Another Hexagonal Representation using $O(n) \times O(n)$ Area}

In this section, we present an alternative approach to proving Theorem 1 1 . This approach is based on Kant's algorithm for hexagonal grid drawings of 3-connected, 3 -regular planar graphs [20]. Although the modification needed is direct, we feel that our previous approach is a more intuitive and constructive technique that yields better fundamental insight into the nature of the problem.

In Kant's algorithm the drawing is obtained by looking at the dual graph and processing its vertices in the canonical order. In the final drawing, however, there are two non-convex faces, separated by an edge not drawn as a straight-line segment. We address these problems by adding some extra vertices in a pre-processing step. Once the dual of this augmented graph is embedded, the faces corresponding to the extra vertices can be removed to yield the desired $O(n) \times O(n)$ grid drawing.

Let $H=(V, E)$ be a 3-connected, 3-regular planar graph. Note that the dual $D(H)$ is fully triangulated, as each face in the dual corresponds to exactly one vertex in $H$. So, for $f$ faces in $H$, we have $f$ vertices in $D(H)$. We first compute a canonical ordering on the vertices of $D(H)$ as defined by de Fraysseix et al. [8]. Let $v_{1}, \ldots, v_{f}$ be the vertices in $D(H)$ in this canonical order.

Kant's algorithm now constructs a drawing for $H$ on the hexagonal grid such that all edges but one have slopes $0^{\circ}, 60^{\circ}$ or $-60^{\circ}$, with the one edge with bends lying on the outer face. The typical structure of those drawings is shown in Figure 9(a). Although we focus our description using the hexagonal grid, to place on the rectilinear grid, the corresponding slopes are $0^{\circ}, 90^{\circ}$ and $-45^{\circ}$.

The algorithm incrementally constructs the drawing by adding the faces of $H$ in reverse order of the canonical order of the corresponding vertices in $D(H)$. We let $w_{i}$ be the vertices of $H$. Let face $F_{i}$ correspond to vertex $v_{i}$ in $D(H)$. The algorithm starts with a triangular region for the face $F_{f}$ that corresponds to vertex $v_{f}$. The vertex $w_{x}$ that is adjacent to $F_{f}, F_{1}$ and $F_{2}$ is placed at the bottom. Let $w_{y}$ and $w_{z}$ 


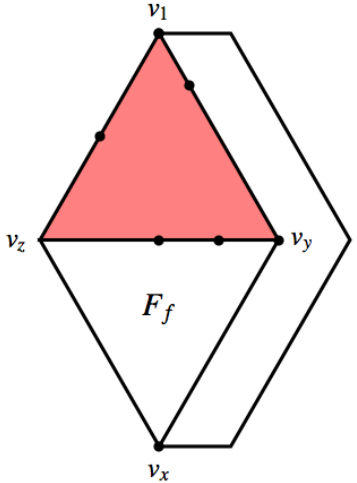

(a)

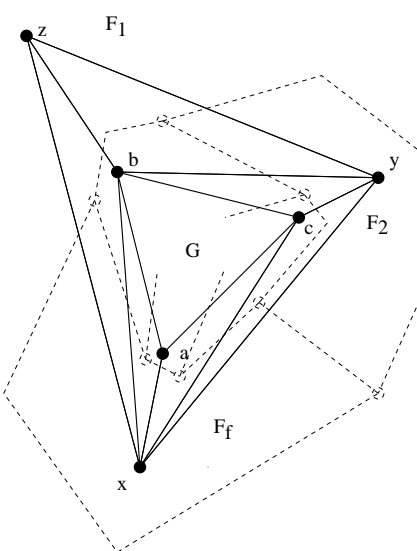

(b)

Fig. 9 (a) Polygonal structure obtained from Kant's algorithm. (b) Graph $G$ augmented by vertices $x$, $y$ and $z$ together with its dual which serves as the input graph for Kant's algorithm.

be the neighbors of $w_{x}$ in $F_{f}$. These three vertices form the corners of the first face $F_{f} .\left(w_{x}, w_{z}\right)$ and $\left(w_{x}, w_{y}\right)$ are drawn upward with equal lengths and slopes $-\sqrt{3}$ and $\sqrt{3}$, respectively. All the edges on the path between $w_{y}$ and $w_{z}$ along $F_{f}$ are drawn horizontally between the two vertices. From this first triangle, all other faces are added in reverse canonical order to the upper boundary of the drawing region. If a face is completed by only one vertex $w_{i}$, this vertex is placed appropriately above the upper boundary such that it can be connected by two edges with slopes $-\sqrt{3}$ and $\sqrt{3}$, respectively. If the face is completed by a path, then the two end segments of the path have slopes $-\sqrt{3}$ and $\sqrt{3}$, while the other edges are horizontal. The construction ends when $w_{1}$ is inserted, corresponding to the outer face $F_{1}$. Note that there is an edge between $w_{1}$ and $w_{x}$, which is drawn using some bends. This edge is adjacent to the faces $F_{1}$ (the outer face) and $F_{2}$.

From this construction, we can observe that the angles at faces $F_{f}, \ldots, F_{3}$ have size $\leq 180^{\circ}$ as the first two edges do not enter the vertex from above, and the last edge leaves the vertex upwards. Hence, we have the following result.

Lemma 6 The faces $F_{f}, \ldots, F_{3}$ are convex, and as the slopes of the edges are $\pm \sqrt{3}$ or 0 , they are drawn with at most six sides.

This property is exactly what we are aiming for, as the vertices of our input graph $G$ should be represented by convex regions of at most six sides. Unfortunately, Kant's algorithm creates two non-convex faces $F_{1}$ and $F_{2}$ separated by an edge which is not drawn as a line segment. Furthermore, the face $F_{f}$ is drawn as large as all the remaining faces $F_{3}, \ldots, F_{f-1}$ together.

Kant gave an area estimate for the result of his algorithm which is the same for both hexagonal and rectilinear grids. A corollary of Kant's algorithm is the following:

Corollary 2 For a given 3-connected, 3-regular planar graph $H$ of $n$ vertices, $H-w_{x}$ can be drawn within an area of $n / 2-1 \times n / 2-1$. 
To apply Kant's result to the problem of constructing a touching hexagons representation, we enlarge the embedded input graph $G$ so that the dual of the resulting graph $G^{\prime}$ can be drawn using Kant's algorithm in such a way that the original vertices of $G$ correspond to the faces $F_{3}, \ldots, F_{f-1}$.

We add 3 vertices corresponding to faces $F_{1}, F_{2}$ and $F_{f}$ in Kant's algorithm. Since $G$ is fully triangulated, let $a, b$ and $c$ be the vertices at the outer face of $G$ in clockwise order. We add the vertices $x, y$ and $z$ in the outer face and connect to $G$ so that $z$ corresponds to the outer face $F_{1}, y$ to $F_{2}$, and $x$ to $F_{f}$. First, we add $x$ and connect it to $a, b$ and $c$ such that $b$ and $c$ are still in the outer face. Then we add $y$ and connect it to $x, b$ and $c$ such that $b$ is still in the outer face. Finally, we add $z$ and connect it to $x, y$ and $b$ such that $x, y$ and $z$ now form the outer face; see Figure 9 (b).

Since the vertices $x, y$ and $z$ are on the outer face, we can choose which one is first, second and last in the canonical order. We then apply Kant's algorithm with the canonical order $v_{1}=z, v_{2}=y$ and $v_{f}=x$. After construction, we remove the regions corresponding to vertices $x, y$ and $z$, yielding a hexagonal representation of $G$.

Given any (connected) planar graph $G$, we can make it fully triangulated using the technique described in Section 3.1. We can then remove the added vertices and edges. Since Kant's algorithm runs in linear time, and our emendations can be done in linear time, we get another proof for Theorem 1 . We again use at most three slopes for each representation with sides having slopes $\pm \sqrt{3}$ or 0 (or $0,+\infty$ and -1 ).

For a triangulated input graph $G=(V, E)$, we have $n$ vertices and, by Euler's formula, $2 n-4$ faces. Since we enhanced our graph to $n+3$ vertices, we have $f=2 n+2$ faces. Those faces are the vertices in the dual $D(G)$ which is the input to Kant's algorithm. His area estimation gives an area of $f / 2-1 \times f / 2-1$ for $f$ vertices when we coalesce the faces $F_{1}, F_{2}$ and $F_{f}$ into a single outer face by removing the corresponding vertices and edges. Thus, we get an area bound of $n \times n$ using exactly the same argument as in [20].

\section{Conclusion and Future Work}

Thomassen [31] had shown that not all planar graphs can be represented by touching pentagons, where the external boundary of the figure is also a pentagon and there are no holes. Our results are more general, as we do not insist on the external boundary being a pentagon or on there being no holes between pentagons. It is possible to derive algorithms for convex hexagonal representations for general planar graphs from several earlier papers, e.g., de Fraysseix et al. [8], Thomassen [31], and Kant [20]. However, these do not immediately lead to algorithmic solutions to the problem of computing a graph representation with convex low-complexity touching polygons. To the best of our knowledge, this problem has never been formally considered.

In this paper, we presented several results about touching $k$-sided graphs. We showed that, for general planar graphs, six sides are necessary and sufficient, and that the algorithm for creating a touching hexagons representation can be modified to yield an $O(n) \times O(n)$ drawing area. Finally, we discussed a different algorithm for general planar graphs which yields a similar drawing area. 
Several interesting related problems are open. What is the complexity of deciding whether a given planar graph can be represented by touching triangles, quadrilaterals, or pentagons? In the context of rectilinear cartograms, the vertex-weighted problem has been carefully studied. However, the same problem without the rectilinear constraint has received less attention. Finally, it would be interesting to characterize the subclasses of planar graphs that allow for touching triangles, touching quadrilaterals, and touching pentagons representations.

Acknowledgements We would like to thank Therese Biedl for pointing out the very relevant work by Kant and Thomassen and the anonymous referees for their helpful and thoughtful comments.

\section{References}

1. M. J. Alam, T. Biedl, S. Felsner, M. Kaufmann, and S. G. Kobourov. Proportional contact representations of planar graphs. Tech. Rep. CS 2011-11, 2011, http://www.cs. uwaterloo.ca/research/ $\operatorname{tr} / 2011 / \mathrm{CS}-2011-11 . \mathrm{pdf}$

2. G. D. Battista, W. Lenhart, and G. Liotta. Proximity drawability: A survey. Proc. Graph Drawing, pp. 328-39. Springer-Verlag, Lecture Notes in Computer Science 894, 1994.

3. M. de Berg, E. Mumford, and B. Speckmann. On rectilinear duals for vertex-weighted plane graphs. Discrete Mathematics 309(7):1794-1812, 2009.

4. T. Biedl, A. Bretscher, and H. Meijer. Rectangle of influence drawings of graphs without filled 3cycles. Proc. 7th Int'l. Symp. on Graph Drawing '99, pp. 359-368, 1999.

5. M. Bruls, K. Huizing, and J. J. van Wijk. Squarified treemaps. Proc. Joint Eurographics/IEEE TVCG Symp. Visualization, VisSym, pp. 33-42, 2000.

6. A. L. Buchsbaum, E. R. Gansner, C. M. Procopiuc, and S. Venkatasubramanian. Rectangular layouts and contact graphs. ACM Transactions on Algorithms 4(1), 2008.

7. M. Chrobak and T. Payne. A linear-time algorithm for drawing planar graphs. Inform. Process. Lett. 54:241-246, 1995

8. H. de Fraysseix, P. O. de Mendez, and P. Rosenstiehl. On triangle contact graphs. Combinatorics, Probability and Computing 3:233-246, 1994.

9. H. de Fraysseix, J. Pach, and R. Pollack. Small sets supporting Fary embeddings of planar graphs. Procs. 20th Symposium on Theory of Computing (STOC), pp. 426-433, 1988.

10. H. de Fraysseix, J. Pach, and R. Pollack. How to draw a planar graph on a grid. Combinatorica 10(1):41-51, 1990.

11. K. R. Gabriel and R. R. Sokal. A new statistical approach to geographical analysis. Systematic Zoology 18:54-64, 1969.

12. E. Gansner, Y. Hu, and S. Kobourov. On touching triangle graphs. Graph Drawing, pp. 250-261. Springer Berlin / Heidelberg, Lecture Notes in Computer Science 6502, 2011, http://dx. doi. org/10.1007/978-3-642-18469-7_23 10.1007/978-3-642-18469-7_23.

13. D. Gonçalves, B. Lévêque, and A. Pinlou. Triangle contact representations and duality. Graph Drawing, pp. 262-273. Springer Berlin / Heidelberg, Lecture Notes in Computer Science 6502, 2011, http://dx.doi.org/10.1007/978-3-642-18469-7_24 10.1007/978-3-642-18469-7_24.

14. X. He. On finding the rectangular duals of planar triangular graphs. SIAM Journal of Computing 22(6):1218-1226, 1993

15. X. He. On floor-plan of plane graphs. SIAM Journal of Computing 28(6):2150-2167, 1999.

16. P. Hliněný. Classes and recognition of curve contact graphs. J. Combin. Theory (B) 74(1):87-103, 1998.

17. P. Hliněný and J. Kratochvíl. Representing graphs by disks and balls (a survey of recognitioncomplexity results). Discrete Mathematics 229(1-3):101-24, 2001.

18. J. Hopcroft and R. E. Tarjan. Efficient planarity testing. Journal of the ACM 21(4):549-568, 1974.

19. J. W. Jaromczyk and G. T. Toussaint. Relative neighborhood graphs and their relatives. Proceedings of the IEEE 80:1502-17, 1992.

20. G. Kant. Hexagonal grid drawings. 18th Workshop on Graph-Theoretic Concepts in Computer Science, pp. 263-276, 1992. 
21. G. Kant. Drawing planar graphs using the canonical ordering. Algorithmica 16:4-32, 1996. (special issue on Graph Drawing, edited by G. Di Battista and R. Tamassia).

22. G. Kant and X. He. Regular edge labeling of 4-connected plane graphs and its applications in graph drawing problems. Theoretical Computer Science 172:175-93, 1997.

23. P. Koebe. Kontaktprobleme der konformen Abbildung. Berichte über die Verhandlungen der Sächsischen Akademie der Wissenschaften zu Leipzig. Math.-Phys. Klasse 88:141-164, 1936.

24. K. Koźmiński and W. Kinnen. Rectangular dualization and rectangular dissections. IEEE Transactions on Circuits and Systems 35(11):1401-16, 1988.

25. Y.-T. Lai and S. M. Leinwand. Algorithms for floorplan design via rectangular dualization. IEEE Transactions on Computer-Aided Design 7:1278-89, 1988.

26. Y.-T. Lai and S. M. Leinwand. A theory of rectangular dual graphs. Algorithmica 5:467-83, 1990.

27. C.-C. Liao, H.-I. Lu, and H.-C. Yen. Compact floor-planning via orderly spanning trees. Journal of Algorithms 48:441-451, 2003.

28. G. Liotta, A. Lubiw, H. Meijer, and S. H. Whitesides. The rectangle of influence drawability problem. Computational Geometry: Theory and Applications 10:1-22, 1998.

29. M. Rahman, T. Nishizeki, and S. Ghosh. Rectangular drawings of planar graphs. Journal of Algorithms 50(1):62-78, 2004

30. P. Steadman. Graph-theoretic representation of architectural arrangement. The Architecture of Form, pp. 94-115. Cambridge University Press, 1976.

31. C. Thomassen. Plane representations of graphs. Progress in Graph Theory, pp. 43-69, 1982.

32. C. Thomassen. Interval representations of planar graphs. J. Combin. Theory (B) 40:9-20, 1988.

33. J. Q. Walker, II. A node-positioning algorithm for general trees. Software: Practice and Experience 20(7), 1990.

34. G. K. Yeap and M. Sarrafzadeh. Sliceable floorplanning by graph dualization. SIAM Journal on Discrete Mathematics 8(2):258-80, 1995. 
A Examples of Touching Hexagons Graph Drawings

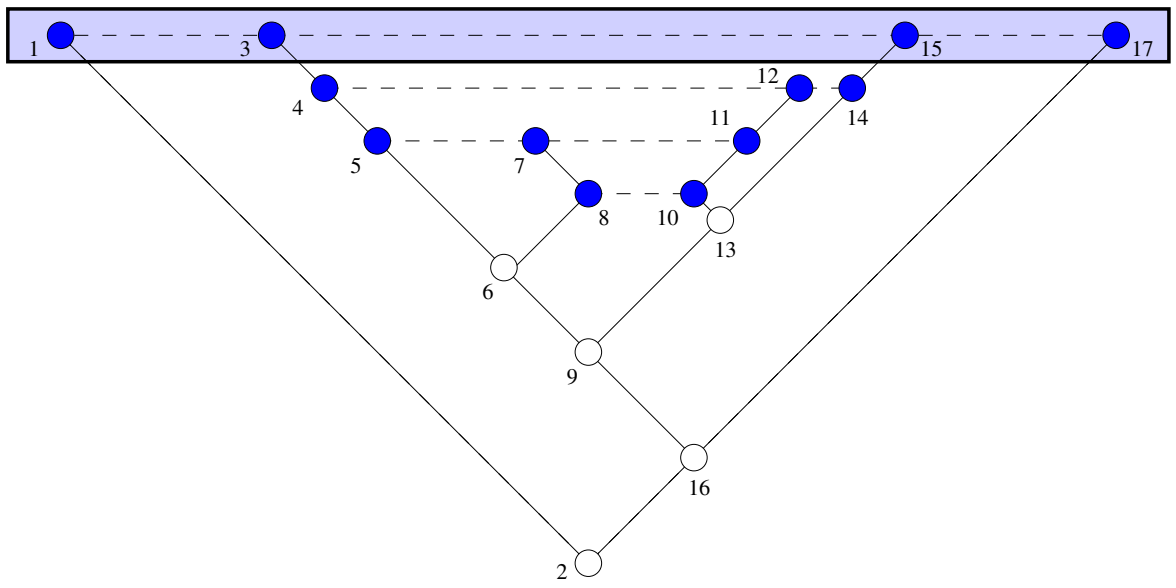

(a)

\begin{tabular}{|c|c|c|c|c|c|}
\hline Node & $x$ & $y$ & shift & $\mathrm{h}$ & Steps \\
\hline 1 & 0 & 0 & 0 & 0 & $2 \mathrm{a}$ \\
3 & 0 & 0 & 0 & 0 & $2 \mathrm{a}$ \\
15 & 0 & 0 & 0 & 0 & $2 \mathrm{a}$ \\
17 & 0 & 0 & 0 & 0 & $2 \mathrm{a}$ \\
\hline
\end{tabular}

(b)

Fig. 10 Example execution of the compaction algorithm on the graph and subsequent capped binary tree $G_{T}$ from Figure 6 (a) The tree with the initial active front set $F$ highlighted. Observe that 7 and 12 are leaf nodes but are not in $F$ because $(5,7,11)$ and $(4,12,14)$ are not all leaves in the current working tree $G_{C}=G_{T}$. (b) The table of the nodes altered in the current iteration of the algorithm indicating the $x, y$, and shift values for each node along with the specific steps applied to determine these new values. 


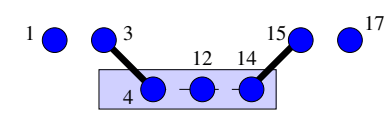

\begin{tabular}{|c|c|c|c|c|c|}
\hline Node & $x$ & $y$ & shift & $\mathrm{h}$ & Steps \\
\hline 4 & 1 & -1 & 0 & 1 & $2 \mathrm{~b}, 3 \mathrm{~b}$ \\
12 & 0 & -1 & 0 & 1 & $2 \mathrm{a}, 3 \mathrm{a}$ \\
14 & -1 & -1 & 0 & 1 & $2 \mathrm{~b}, 3 \mathrm{~b}$ \\
\hline
\end{tabular}

(a)

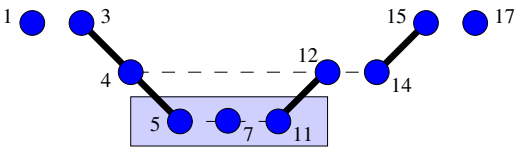

\begin{tabular}{|c|c|c|c|c|c|}
\hline Node & $x$ & $y$ & shift & $\mathrm{h}$ & Steps \\
\hline 5 & 2 & -2 & 0 & 2 & $2 \mathrm{~b}, 3 \mathrm{~b}$ \\
7 & 0 & -2 & 0 & 2 & $2 \mathrm{a}, 3 \mathrm{a}$ \\
11 & -1 & -2 & 0 & 2 & $2 \mathrm{~b}, 3 \mathrm{~b}$ \\
\hline
\end{tabular}

(b)

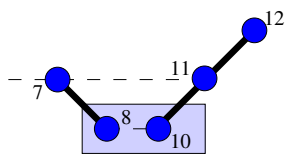

\begin{tabular}{|c|c|c|c|c|c|}
\hline Node & $x$ & $y$ & shift & $\mathrm{h}$ & Steps \\
\hline 8 & 1 & -3 & 0 & 3 & $2 \mathrm{~b}, 3 \mathrm{a}$ \\
10 & -2 & -3 & 0 & 3 & $2 \mathrm{~b}, 3 \mathrm{a}$ \\
\hline
\end{tabular}

(c)

Fig. 11 The next three iterations of subsequent active fronts. For space only a portion of the tree is shown. (a) The active front consisting of nodes 4,12 , and 14 . Observe that all three nodes are placed (by step 3) at the lowest $y$-value. Thus, node 12's $y$ position is set to -1 . (b) The active front consisting of nodes 5,7 , and 11. (c) The active front consisting of nodes 8 and 10. 


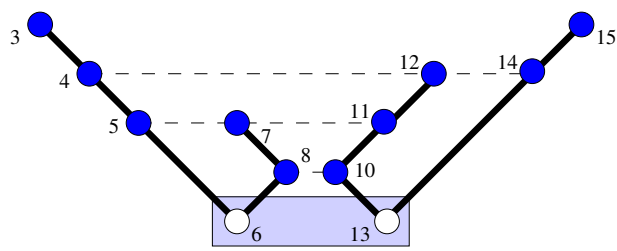

\begin{tabular}{|c|c|c|c|c|c|}
\hline Node & $x$ & $y$ & shift & $\mathrm{h}$ & Steps \\
\hline 6 & 4 & -4 & 0 & - & $2 \mathrm{c}$ \\
13 & -1 & -4 & 0 & - & $2 \mathrm{c}$ \\
\hline \hline 8 & 1 & -3 & 4 & - & $2 \mathrm{c}$ \\
14 & -1 & -1 & 3 & - & $2 \mathrm{c}$ \\
\hline
\end{tabular}

(a)

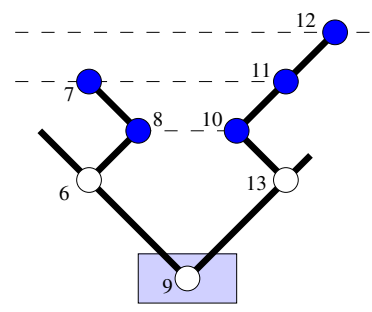

\begin{tabular}{|c|c|c|c|c|c|}
\hline Node & $x$ & $y$ & shift & $\mathrm{h}$ & Steps \\
\hline 9 & 6 & -6 & 0 & - & 2c \\
\hline \hline 13 & -1 & -4 & 9 & - & 2c \\
\hline
\end{tabular}

(b)

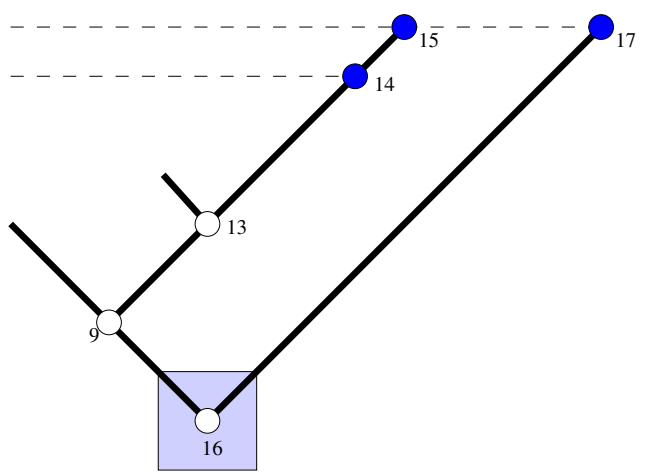

\begin{tabular}{|c|c|c|c|c|c|}
\hline Node & $x$ & $y$ & shift & $\mathrm{h}$ & Steps \\
\hline 16 & 7 & -7 & 0 & - & $2 \mathrm{c}$ \\
\hline \hline 17 & 0 & 0 & 14 & - & $2 \mathrm{c}$ \\
\hline
\end{tabular}

(c)

Fig. 12 The next three iterations of subsequent active fronts. In these examples, there are no cap nodes involved so Step 3 is not executed. (a) The active front consisting of nodes 6 and 13. Nodes 8 and 14 are affected because they are shifted as part of the compaction step (2c). (b) The active front consisting of node 9 . Node 13 is shifted in Step $2 \mathrm{c}$ because it is the root of the right subtree of node 9. (c) The active front consisting of node 16. Node 17 is shifted in Step 2c because it is the root of the right subtree of 16 . 


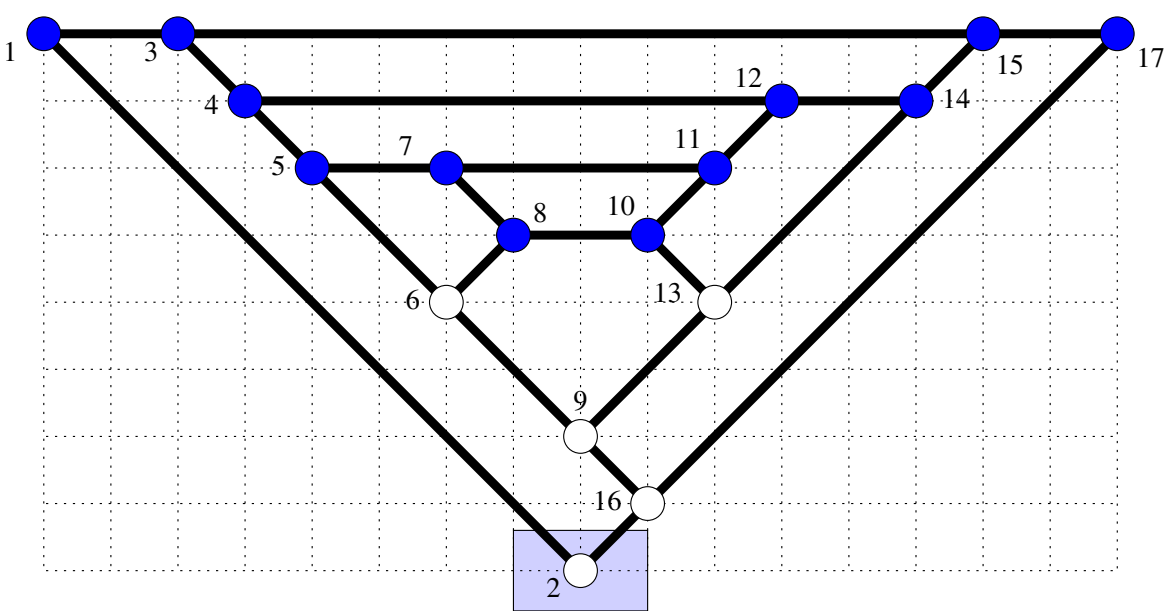

(a)

\begin{tabular}{|c|c|c|c|c|c|}
\hline Node & $x$ & $y$ & shift & h & Steps \\
\hline 2 & 8 & -8 & 0 & - & 2c \\
\hline \hline 16 & 7 & -7 & 2 & - & 2c \\
\hline
\end{tabular}

(b)

\begin{tabular}{|c|c|c|c|}
\hline Node & $x$ & $y$ & shift \\
\hline 2 & 8 & -8 & 0 \\
1 & 0 & 0 & 0 \\
16 & 9 & -7 & 2 \\
9 & 8 & -6 & 2 \\
6 & 6 & -4 & 2 \\
5 & 4 & -2 & 2 \\
4 & 3 & -1 & 2 \\
3 & 2 & 0 & 2 \\
8 & 7 & -3 & 6 \\
7 & 6 & -2 & 6 \\
13 & 10 & -4 & 11 \\
10 & 9 & -3 & 11 \\
11 & 10 & -2 & 11 \\
12 & 11 & -1 & 11 \\
14 & 13 & -1 & 14 \\
15 & 14 & 0 & 14 \\
17 & 16 & 0 & 16 \\
\hline
\end{tabular}

(c)

Fig. 13 The final iteration along with the propagation of the shifts to the children. (a) The final drawing (b) The table of the two affected nodes by the last step. (c) The final table of all node positions including the shift values applied after propagation, listed in preorder traversal of the tree. 

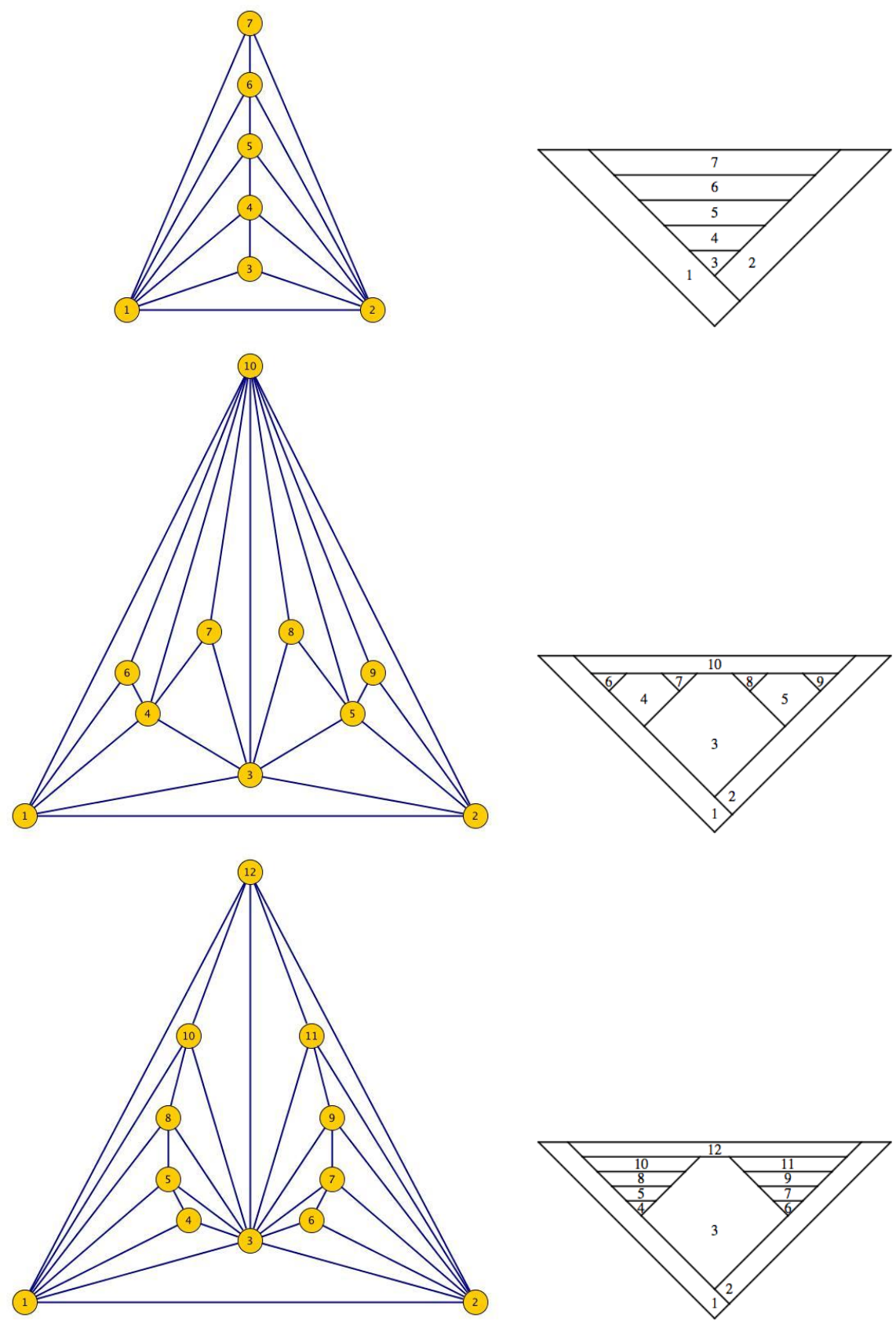

Fig. 14 Examples illustrating input graphs and their corresponding touching hexagons representations. 